\title{
Ultrasonographic Detection of Airway Obstruction in a Model of Obstructive Sleep Apnea
}

\section{(ㅇ)(우)(}

Authors

Amal Isaiah', Reuben Mezrich², Jeffrey Wolf ${ }^{3}$

Affiliations

1 Otorhinolaryngology - Head and Neck Surgery, University of Maryland School of Medicine, Baltimore, United States

2 Radiology, University of Maryland School of Medicine, Baltimore, United States

3 Otolaryngology - Head and Neck Surgery, University of Maryland School of medicine, Baltimore, United States

Key words

head/neck, ultrasound, segmentation, technical aspects

received 19.07.2016

revised $\quad 06.12 .2016$

accepted 08.12.2016

Bibliography

DOI http://dx.doi.org/10.1055/s-0042-124503

Ultrasound Int Open 2017; 3: E34-E42

(c) Georg Thieme Verlag KG Stuttgart · New York

ISSN 2199-7152

Correspondence

Dr. Jeffrey Wolf, MD

Otorhinolaryngology - Head and Neck Surgery

University of Maryland School of Medicine

16 S Eutaw St Ste 500

Baltimore, MD 21201

United States

Tel.: + 1/410/328 6866, Fax: +1/410/328 5852

jwolf@som.umaryland.edu

\section{ABSTRACT}

Purpose Obstructive sleep apnea (OSA) is a common clinical disorder characterized by repetitive airway obstruction during sleep. The gold standard for diagnosis of OSA, polysomnogram (PSG), cannot anatomically localize obstruction. Precise identification of obstruction has potential to improve outcomes following surgery. Current diagnostic modalities that provide this information require anesthesia, involve ionizing radiation or disrupt sleep. To mitigate these problems, we conceived that ultrasound (US) technology may be adapted (i) to detect, quantify and localize airway obstruction and (ii) for translational application to home-based testing for OSA.

Materials and Methods Segmental airway collapse was induced in 4 fresh cadavers by application of negative pressure. Following visualization of airway obstruction, a rotary US probe was used to acquire transcervical images of the airway before and after induction of obstruction. These images $(n=800)$ were analyzed offline using image processing algorithms.

Results Our results show that the non-obstructed airway consistently demonstrated the presence of a US air-tissue interface. Importantly, automated detection of the air-tissue interface strongly correlated with manual measurements. The algorithm correctly detected an air-tissue interface in $90 \%$ of the US images while incorrectly detecting it in $20 \%$ (area under the curve $=0.91$ ).

Conclusion The non-invasive detection of airway obstruction using US represents a major step in expanding OSA diagnostics beyond PSG. The preliminary data obtained from our model could spur further research in non-invasive localization of obstruction. US offers the benefit of precise localization of the site of obstruction, with potential for improving outcomes in surgical management

\section{Introduction}

Obstructive sleep apnea (OSA) is a common clinical disorder characterized by paroxysmal and repetitive episodes of partial or complete airway obstruction that occur during sleep [1]. Homeostatic dysregulation resulting from chronic sleep deprivation imparts increased risk for cardiovascular [2], metabolic [3] and neurobehavioral [4] problems as well as a significant number of sudden deaths [5]. Large population-based epidemiologic studies have highlighted the high prevalence and wide spectrum of the severity of OSA, and importantly, that even mild OSA may be associated with significant morbidity [6]. Consequently, timely diagnosis of OSA is important to halt this cascade of events, leading to an increasing interest in secondary prevention using diagnostic tests.

A polysomnogram (PSG), also called a sleep study, is the universally accepted gold standard for the diagnosis and stratification of OSA [7]. The principal metrics derived from a PSG that are indica- tive of the frequency and severity of airway obstruction are routinely used for clinical decision-making including surgical treatment. However, with increasing costs and a growing supply-demand mismatch, a rising number of PSGs appear to have been supplanted by diagnostic tools used within the context of out-oflab testing (OLT). Consequently, novel techniques that span wristworn sensors [8] to abbreviated nocturnal oximetry [9] have shown considerable promise recently.

Notwithstanding their high sensitivity, neither in-lab PSG nor OLT can directly visualize anatomic details within the airway-information that may be crucial in predicting outcomes following surgery performed to ameliorate airway obstruction [10,11]. Additionally, identification of the site(s) of airway collapse by itself can be used to diagnose obstruction sleep apnea. Although imaging technologies such as computed tomography $(\mathrm{CT})$, magnetic resonance imaging (MRI) and cephalometry $[12,13]$ are readily availa- 
ble, the use of these methods is generally restricted and not feasible for most patients. The possible reasons may be related to concerns for exposure to ionizing radiation, costs and the relative limitations on obtaining real-time dynamic imaging during natural sleep within an image scanner. Importantly, the physiology of sleep in its natural state may be altered by the administration of anesthetic agents, making accurate diagnosis from drug-induced sleep imaging less feasible.

One imaging technology that has not been widely harnessed to diagnose airway obstruction in OSA is ultrasound. As a diagnostic modality, it is inexpensive, portable, quiet, widely available and does not involve the use of ionizing radiation [14]. Overall trends demonstrate the increasing use of cross-sectional imaging of the neck [15]. Ultrasound technology has been previously utilized to demonstrate the structural anatomy of the upper airway and trachea [16] with good correlation observed when compared with MRI-derived soft tissue anatomy [17].

More recently, submental ultrasonography has been used to predict the severity of sleep apnea by measuring tongue base thickness [18]. Shu et al. [19] demonstrated its utility in quantifying airway obstruction in clinical volunteers with polysomnographically determined severe OSA. This method established nearly $100 \%$ sensitivity in demonstrating retropalatal soft tissue collapse following application of the Müller maneuver. In this study, the authors used ultrasound-derived air-tissue interfaces to demarcate anatomic boundaries. Although the study has significant implications, the technology has not been explored further for dynamic detection of airway obstruction.

The interaction of ultrasound with an air-tissue interface - in which the high acoustic impedance of air results in near-complete reflection of an incident ultrasonic wavefront from a transducer [20] - may be used to significant advantage in the functional detection of airway collapse. To validate this technology, we have developed a unique cadaver-based model of upper airway obstruction, in which we corroborate endoscopic visualization with air-tissue interfaces generated by a rotary ultrasound probe detecting all areas of potential upper airway obstruction nearly simultaneously. In addition, we have developed a novel ultrasound-based image processing algorithm that provides the significant added benefit of (i) automation that allows scanning of a large continuous series of images, and (ii) demonstration of the exact site of obstruction in a noninvasive, inexpensive and portable footprint. Used alone (or in combination with in-lab polysomnography), this technique has potential to both diagnose OSA, and accurately identify the site(s) of obstruction in patients who may benefit from precise localization prior to OSA surgery.

\section{Methods}

\section{Cadaver model of airway obstruction in OSA}

4 fresh unfixed cadavers ( 2 male and 2 females) procured from the State Anatomy Board were utilized for this study. As only anonymized cadavers were used, the study was not within the purview of the institutional review board. Prior to the study, we confirmed that there were no direct airway interventions-except endotracheal intuba- tion - performed in any of the cadavers that could potentially impact the results obtained from this study.

Following supine positioning, a low tracheostomy incision was made at the level of the third tracheal ring. Subcutaneous fat was dissected away. The anterior wall of the trachea was identified and a tracheal window was completed. A size 8 disposable cuffed tracheostomy (DCT ${ }^{\mathrm{TM}}$ Covidien, Minneapolis, MN) tube was inserted with its distal end pointing superiorly ( $\mathbf{F i g .} \mathbf{1 a}, \mathbf{b})$. The position of the tracheostomy tube was confirmed by direct visualization of the appliance tip using a flexible rhinolaryngoscope (ENF-GP) interfaced with a video workstation (Olympus Exera II CLV-180, Tokyo, Japan) inserted via the oral cavity and advanced until the tracheostomy appliance was seen in its expected position ( $\mathbf{F i g}$. 2a). Following satisfactory positioning, the external flanges were secured using 2-point sutures. The cuff around the tube was then inflated to form an effective seal within the airway and the appliance itself was connected to wall-based suction providing negative pressure of $15 \mathrm{~cm}$ of water. This pressure cutoff was chosen based on human physiologic studies that identified critical pharyngeal closing pressures to be in the about $-10 \mathrm{~cm}$ of water [21]. The procedure was repeated in all 4 cadavers. Following application of negative pressure, we confirmed airway collapse endoluminally by endoscopic visualization in all 4 cadavers ( $\triangleright$ Fig. 2 b). Furthermore, the airway reverted to its normal level of patency when suction was released ( $>$ Fig. 2c).

A rotary ultrasonic transducer (3DART 8838, BK Medical, Herlev, Denmark) with its imaging extent spanning a sector of $140^{\circ}$ in the transverse plane was placed horizontally in the submental area slightly above the level of the hyoid bone. The probe specifications allowed imaging in the $6-12 \mathrm{MHz}$ range using a 192-element configuration. All images were obtained using a center frequency of $6 \mathrm{MHz}$ that facilitated image acquisition up to a depth of $10 \mathrm{~cm}$. The transducer was interfaced with an imaging platform (Flex Focus 800, BK Medical, Herlev, Denmark) and skin contact enhanced by the application of conductive gel ( $\boldsymbol{F}$ Fig. 1b). The dynamic range was set to $70 \mathrm{~dB}$. Pilot images were first obtained from the anterior aspect of the neck without application of negative pressure. Airway patency was confirmed following the detection of the air-tissue interface in the posterior third of the neck in all cadavers and correlated visually with endoscopically obtained images. The procedure was repeated in all 4 cadavers, which demonstrated consistent disappearance and appearance of the air-tissue interface with application ( $\triangleright$ Fig. 2 d,e) and subsequent withdrawal of negative pressure ( $\triangleright \mathbf{F i g .} \mathbf{2 d , g}$ ), via the tracheostomy appliance.

Approximately 100 images were obtained from a movie captured at 20 frames/second from each cadaver by scanning the neck by placing the rotational axis of the transducer in the sagittal plane of the cadaver. This plane spanned soft tissues from the skull base to the thoracic inlet, to focus on structures within a range of $6-10 \mathrm{~cm}$ from the skin surface. Each scan was completed in less than $5 \mathrm{~s}$. This matches or improves OSA indices usually determined over $10 \mathrm{~s}$. A similar number of images were acquired from the neck following application of negative pressure to the tracheostomy appliance and induction of airway collapse. A total of 800 images were thus obtained and subsequently saved via the imaging software for analysis offline. 

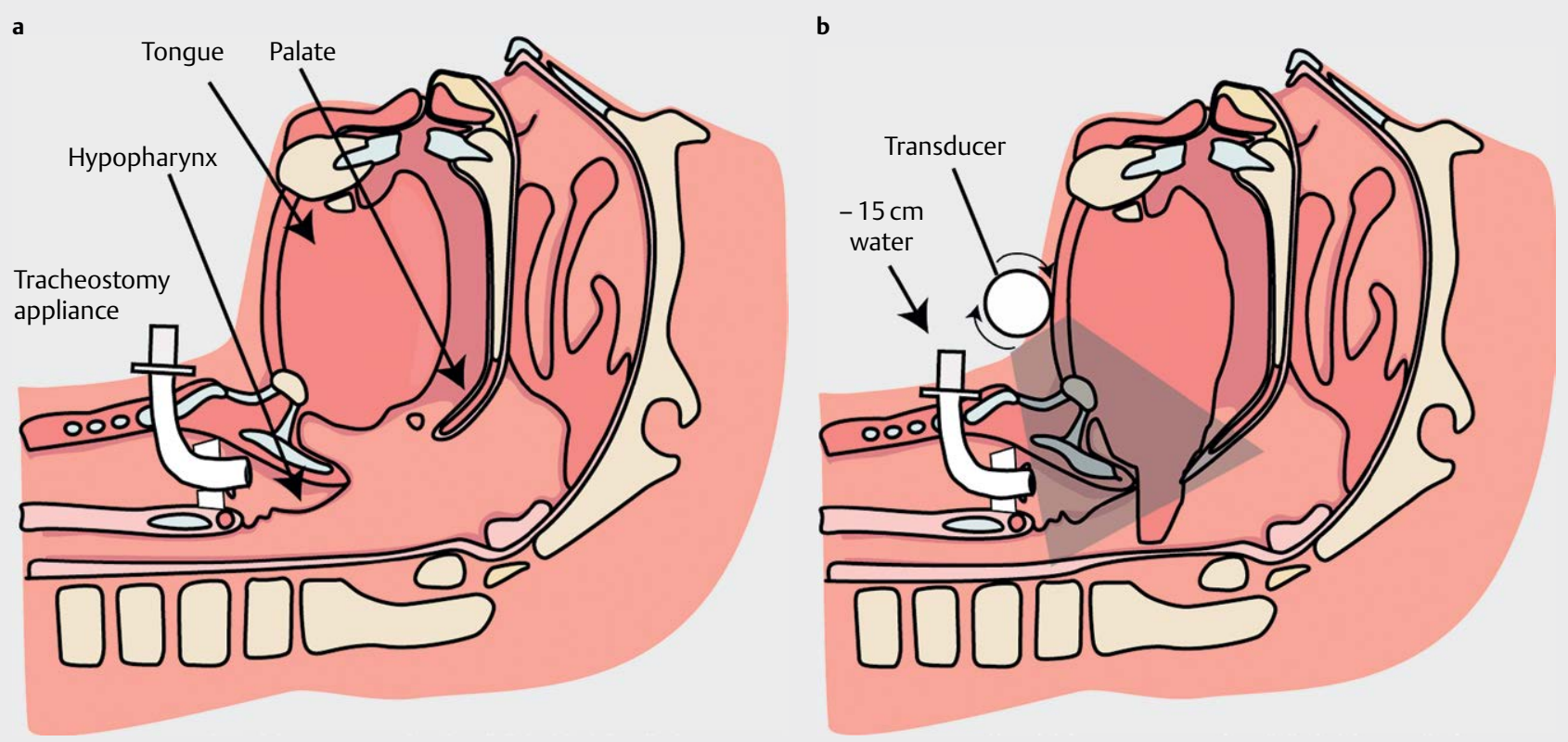

- Fig. 1 Cadaver model of airway obstruction in OSA. a shows surgical placement of a tracheostomy in reverse orientation into the airway. Sites of potential airway obstruction are labelled. $\mathbf{b}$ demonstrates orientation of the ultrasound transducer array placed anteriorly in a submental position and a representative scanning sector. Potential sites of collapse, e. g., at the base of tongue and the palate, are also shown.

\section{Image preparation}

All 800 images were exported after conversion to an 8-bit grayscale format. They were then serially imported to the Image Processing Toolbox ${ }^{\mathrm{TM}}$ associated with the Matlab ${ }^{\circledR}$ program (R2015b, Natick, MA). The images $(n=800)$ were all inspected visually. The air-tissue shadow as the ultrasound wavefront passed through deep neck tissues to enter the airway was observed in all images obtained from the unobstructed cadavers. A region of interest (ROI) was defined within each image corresponding to the approximate location of the air-tissue interface ( $\boldsymbol{F} \mathbf{F i g} \mathbf{2} \mathbf{2} \mathbf{g}, \mathbf{g}$ ). The presence of an air column within each image was confirmed by visual inspection. It is to be noted that the manual inspection of all images was performed by the same individual. The same individual performed delineation of the steps of automated processing of the images as well.

To improve the contrast within each image, an adaptive histogram equalization algorithm was applied. To achieve this, the image was first divided into $8 \times 8$ tiles and a histogram of image intensity obtained from each tile separately. The local histogram within each tile was then interpolated with the distribution from the surrounding tiles, until the whole image was equalized.

Following the adaptive equalization procedure, the region of interest in each image was tagged manually using a polygonal mask to approximate the air-tissue shadow as closely as possible ( $\mathbf{F i g .}$ $\mathbf{2 f}, \mathbf{g})$. The mean pixel intensity was calculated from within the mask and exported. The procedure was then repeated in all 800 images.

\section{Steps of automated image processing}

Each image was initially probed using a linear structuring element to examine the landscape of the image. Specifically, this method was used to scan the image to obtain knowledge of the distribution of pixel intensities in each segment. This step created a sym- metric linear structuring element. The parameters of this step included a length of the structuring element to span 100 pixels, and an angle of $5^{\circ}$ of the line as measured in a counterclockwise direction from the horizontal axis. Step 2 in the same panel consisted of erosion, leading to a reduction in the size of the foreground pixels along the structuring element, thus bringing the background into more prominence. This was followed by morphological reconstruction of the image marker (eroded image) utilizing the original image designated as the mask. Panel b shows the binary image that corresponds to the reconstructed image.

Next, small objects, considered to be artifacts, were removed from the image by a sequence of steps that included (i) creation of a square-shaped structuring element $(5 \times 5)$, (ii) morphological closing using the structuring element as defined in (i) followed by dilation, (iii) removal of small connected components (defined as smaller than 20 pixels in size). Once this operation was applied, the outline of the detected region was further enhanced by a dilation operation utilizing a linear structuring element with a length of 10 pixels.

Visual inspection revealed that the air-tissue interface was always closest to the spine, and hence ubiquitously present in the posterior $40 \%$ of each image. A mask was created subsequently to measure the mean pixel intensity within the air-tissue interface (parameters: horizontal least distance of the mask between 20 and 130 pixels; vertical least distance between 150 and 350 pixels). This was then applied (panel c in $>$ Fig. 3 ) to the original image (panel d). The mean pixel intensity within the region(s) constrained by the thus created mask was calculated and the results exported.

An image was considered obstructed if the mean pixel intensity was less than 150 gray level units. The parameters described above were chosen to have greater than a $90 \%$ agreement rate be- 

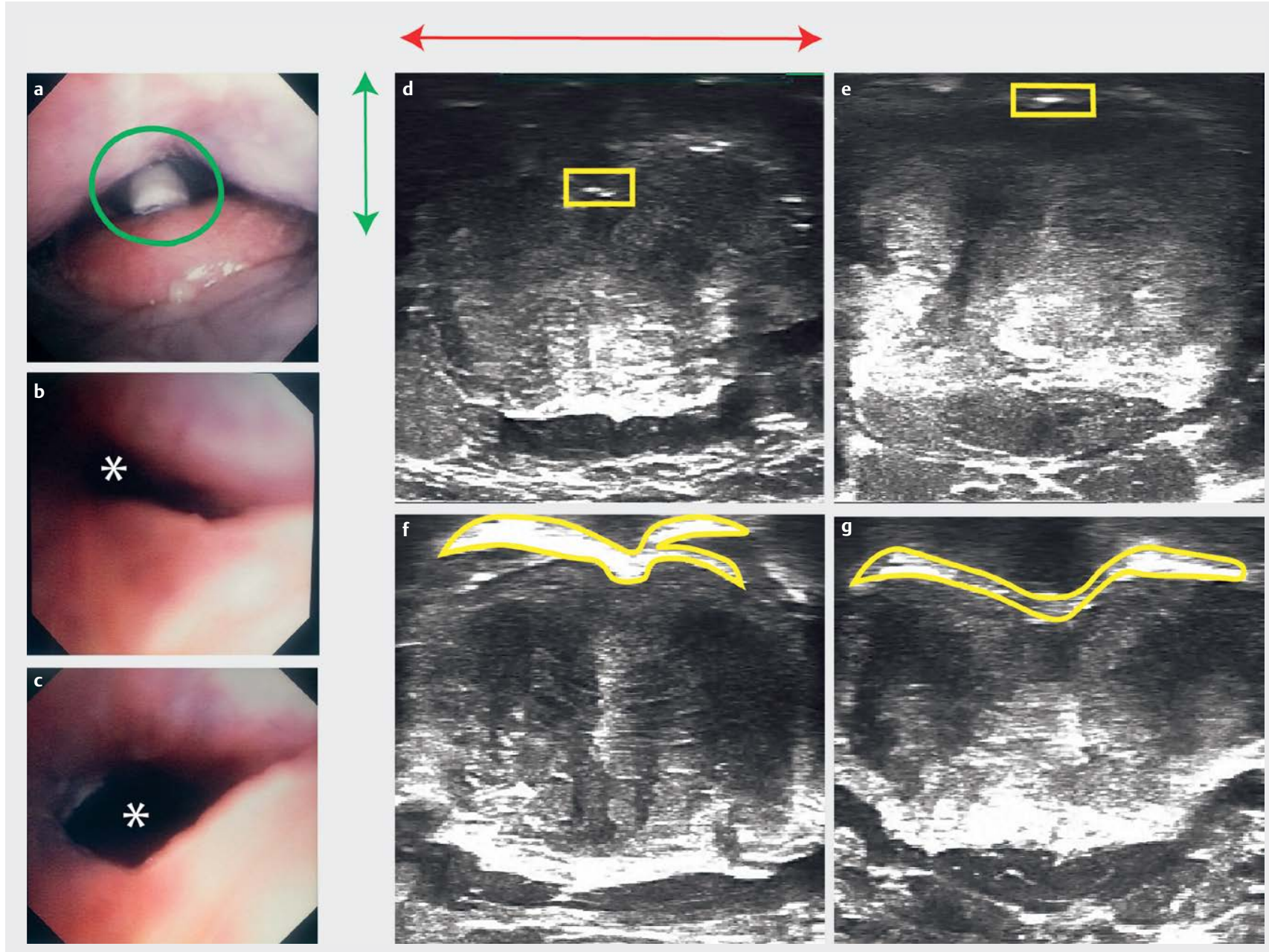

- Fig. 2 Key ultrasonographic images of airway obstruction. a photograph of the base of tongue and the epiglottis with the tip of the tracheostomy appliance highlighted by the green circle. $\mathbf{b}$ endoscopic view of airway collapse induced by application of negative pressure via the tracheotomy conduit with the airway marked by * $\mathbf{c}$ shows the exact same location as $\mathbf{b}$ following release of negative pressure, with subsequent expansion of the airway luminal diameter. $\mathbf{d}$ and $\mathbf{e}$ represent ultrasonographic images acquired transcervically, in 2 different cadavers, after application of negative pressure. Yellow boxes in these images show the attenuated air-tissue interface, compatible with the significantly diminished air column observed in b. The green (vertical extent) and red (horizontal extent) lines outside $\mathbf{d}$ confine the location of this interface in the posterior $40 \%$ of the neck. $\mathbf{f}$ and $\mathbf{g}$ indicate manually determined masks (yellow) marked in yellow, which establish the presence of an air-tissue interface. These also correspond to c wherein an air column is present $\left({ }^{*}\right)$. Together, these images validate the detection of the air-tissue interfaces affected by the application of negative pressure.

tween the status of airway obstruction (obstructed or not) assessed manually and the automated method and subsequently applied to all of the images including those obtained from other cadavers.

\section{Data analysis}

The mean pixel intensity within the manually determined mask and that identified in an automated fashion within each image was compared over the entire dataset using summary measures. The impact of each condition was then analyzed separately. Cumulative probability distribution functions were plotted against all 4 conditions (manual vs. automated, in obstructed vs. unobstructed images, - Fig. 4a). Means and standard deviations were compared using analysis of variance (ANOVA). Multiple comparisons were performed for post-hoc analysis and P-values were calculated. A value less than 0.05 was deemed significant. Conditional correlation was examined by computing Pearson's $R^{2}$ in each condition. The relia- bility of the automated image processing technique was examined using a Bland-Altman plot, and both the reproducibility coefficient and the coefficient of variation were calculated to determine whether the automated method was capable of matching the values obtained from the manual calculations. Finally, a receiver operating characteristic (ROC) curve was plotted from an empiric threshold of mean pixel intensity to determine whether there was equivalence in classifying an image as obstructed or unobstructed in image-to-image comparisons. The area under the curve (AUC) was calculated for estimating the performance of the classifier.

\section{Results}

\section{Summary measures}

The mean pixel intensities in the 2 overall conditions (obstructed vs. unobstructed) when calculated using manual and automated 

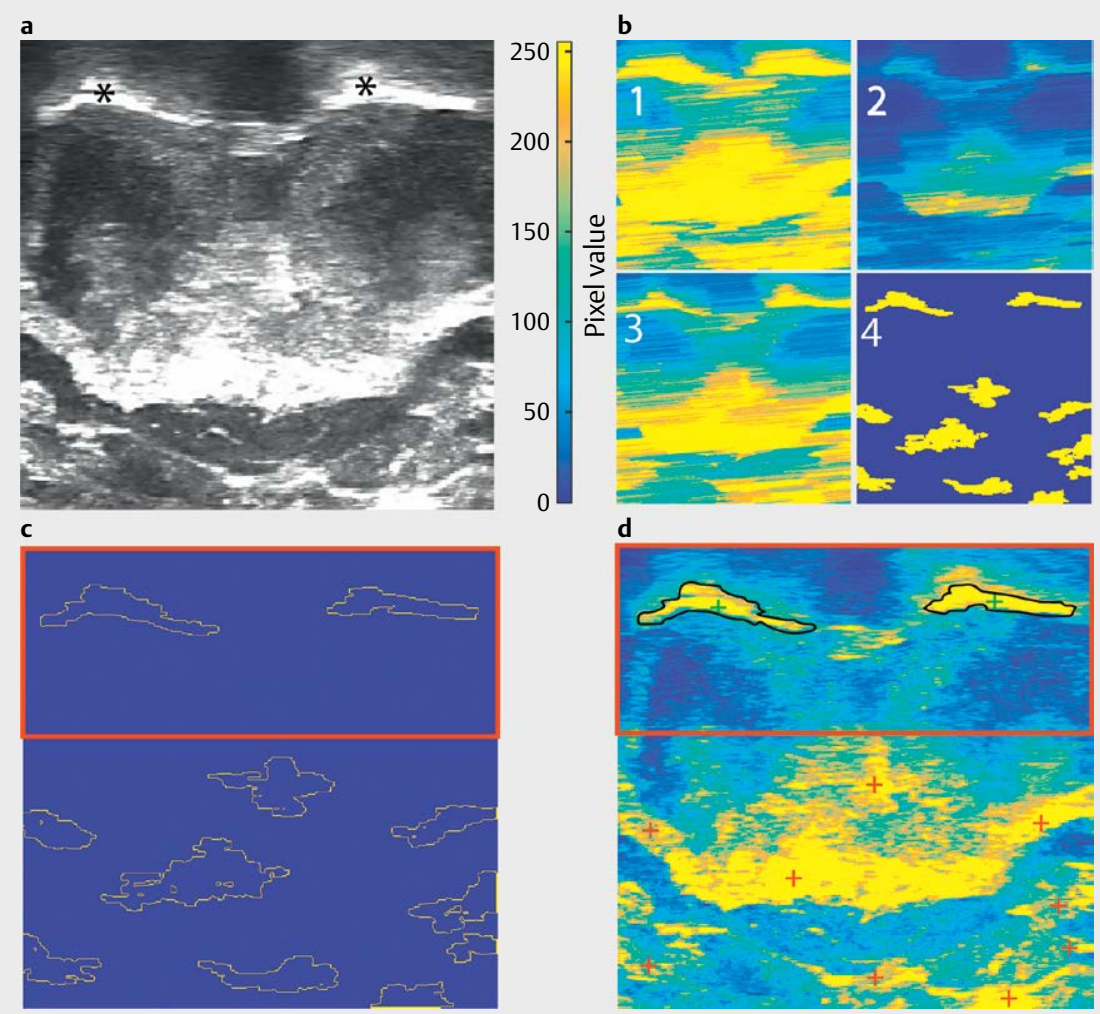

- Fig. 3 Automated detection of air-tissue interface using a combination of morphological operations. A representative image a with a prominent air-tissue interface is first dilated using a structuring element. Asterisks in this sample image highlight a patent airway. This results in enlargement of the boundaries of regions deemed to contain bright pixels within the foreground (b, panel 1). The converse occurs in (2) following erosion, which partially removes the interfaces of bright foreground pixels. Panel 3 is the result of morphological closing. Panel 4 is a binary image, which is the product of a series of steps that include morphological closing and removal of small objects. A boundary detection algorithm applied to the regions in the posterior $40 \%$ of the neck (where airway is anatomically present), highlighted by the red rectangle in c. The mean pixel intensity was calculated within the marked regions and compared to manual estimations. Air-tissue interfaces corresponding to an air column are depicted by green markers $(+)$, while the discarded regions are shown in red. Detailed explanation of these steps is provided in text.

methods were first compared ( $\vee$ Fig. $\mathbf{4 b}$ ). The highest value was observed in the unobstructed condition when the automated method was used (mean \pm SD $=155.1 \pm 34.7$ [obstructed, manual], $162.3 \pm 34.3$ [obstructed, auto], $224.8 \pm 24.8$ [unobstructed, manual] and $233.4 \pm 20.1$ [unobstructed, auto] gray level units, respectively). One-way ANOVA showed that there was a significant difference between these groups $\left(F_{3.1599}=779.1, P<0.001\right)$. Post-hoc multiple comparisons test showed that the automated technique overestimated the mean intensity within each condition $(P<0.05)$, while the obstructed condition also showed overall markedly lower values when estimated using either technique $(P<0.05)$.

Results from comparisons of mean intensity values obtained from a smaller subset of values, i. e., those corresponding to approximate locations of the retropalatal, base of tongue and hypopharyngeal airway segments showed mixed results when each cadaver was considered separately ( $>$ Fig. $\mathbf{5}$ ). In this case, each image series comprising 100 slices was divided into $33 \%$ apiece corresponding to a different airway segment. For example, in cadaver 1 the hypopharyngeal airway (most inferior $33 \%$ ) showed maximal collapse, whereas there was more uniform collapse within all areas in cadaver 2 . There was good concordance between values obtained from the manual and automated methods. However, these values showed an overall increase in the unobstructed condition, regardless of the method of estimation. Statistical comparisons were not performed for the 2 reasons-(i) the exact demarcation of the different segments of the airway could not be determined, and (ii) each cadaver may have different susceptibilities towards collapse in each airway segment and may lead to difficulties in interpretation from the resulting complex data structure.

\section{Classifier performance}

To establish the reliability of the automated classification method, the correlation between automatically and manually estimated mean pixel intensities was examined. Moderate correlation was observed in both obstructed and unobstructed conditions $\left(R^{2}=0.29\right.$ and 0.25 , respectively; $n=400$; one-tailed $P<0.001$ in both conditions). Scatter plots demonstrating these relationships are shown in $>$ Fig. 6a,c.

A Bland-Altman analysis was used to assess the level of agreement between the 2 methods of estimation, which showed the $95 \%$ limits of agreement to range from -58 to 72 gray level units in the obstructed condition and -37 to 54 units in the unobstructed condition. The mean differences in estimation were 7.1 and 8.5 units. The reproducibility coefficients in the 2 conditions were 65 
a

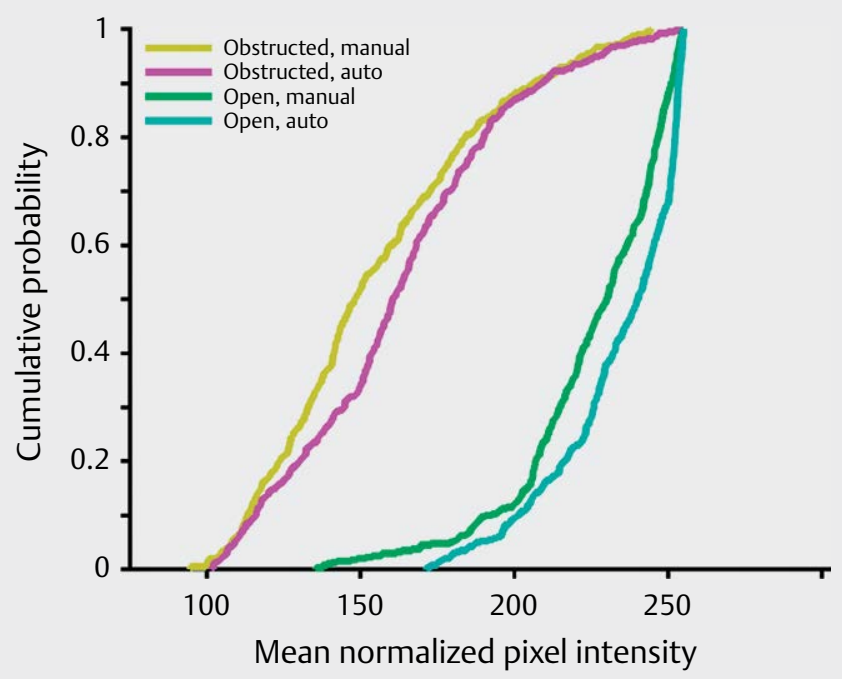

b

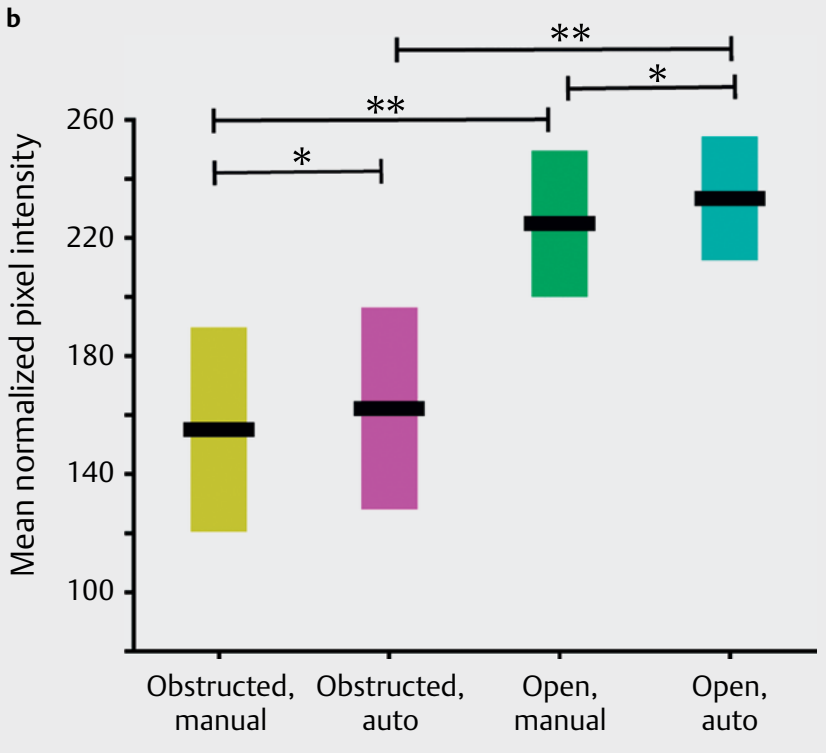

- Fig. 4 Comparison of pixel intensities according to manual or automated detection in 4 main groups. a shows a cumulative probability distribution function highlighting differences between 4 different groups as labelled. Of note, automated estimation of pixel intensities closely approximated results obtained from manual estimation, regardless of the condition (obstructed vs. open). $\mathbf{b}$ boxplots demonstrate differences between the groups. Each distribution within a boxplot is represented by a horizontal black line (median) and the limits highlighted by the extent of the interquartile range between 25 and $75 \%$. The lowest mean pixel intensity was seen in group 1, while the highest was observed in group 4 . Overall, the automated method tended to slightly overestimate the extent of the air-tissue shadow. Also highlighted is the important difference seen between the overall and open groups, which was statistically different. Statistical comparisons are provided in text. ${ }^{*} \mathrm{P}<0.05 ;{ }^{*}{ }^{*} \mathrm{P}<0.001$.

(41\%) and 45 (20\%), respectively. This corresponded to coefficients of variation of 21 and $10 \%$ in the 2 conditions. Scatter plots that explain these differences are shown in $\mathbf{F i g .} \mathbf{6 b}, \mathbf{d}$.

Based on preliminary visual assessments, a mean pixel grayscale intensity value of 150 units was chosen as the cut-off for determining whether a cross-sectional image was obstructed. There were false air-tissue interfaces generated in $12 \%$ of the images, with artifacts generated primarily from air-water interfaces present in preserved cadaveric tissue. A logistical regression model was fitted to the predictor variable (mean pixel intensities of the ROI mask determined from the automated method) and the response variable, which was the assessment whether the image was obstructed based on the cut-off. The fit of the model was statistically significant $\left(X^{2}(400)=531, P<0.0001\right)$ with the model explaining $57 \%$ of the variance. To validate the classifier performance, a receiver operating characteristic curve was constructed ( $\vee$ Fig. 7). In this figure, the true positive rate was plotted against the false positive rate. The area under the curve was calculated as 0.91 , demonstrating that the test was associated with satisfactory performance in determining whether an image is obstructed.

\section{Discussion}

Obstructive sleep apnea is a major determinant of the cost of healthcare in the US and continues to consolidate its presence elsewhere in the world. Thus far, the principal tool used to diagnose OSA is a laboratory-based full-panel PSG. Although there has been increasing interest in other technologies such as home-based oximetry and body-worn sensors, the lack of visualization of the site of obstruction continues to be an unsolved problem. Clearly, real-time identification of segmental airway obstruction would be a valuable component of an ideal diagnostic device. In the absence of a simple noninvasive diagnostic test, methods such as drug-induced sleep endoscopy, cine-MRI and cephalometric indices continue to be used clinically, notwithstanding concerns regarding the use of general anesthesia (i. e., absence of sleep) and exposure to ionizing radiation (when cephalometry is used), as well as significant limitations on generalizability.

Despite the emergence of ultrasound as the gold standard for certain areas of head and neck radiology including thyroid and soft tissue imaging, ultrasound remains an under-utilized modality in OSA diagnostics. It is readily evident that there are clear advantages - e. g., lack of radiation exposure, costs, the absence of ambient noise, ready availability and the ability to perform dynamic imaging. However, the key disadvantages include suboptimal soft tissue resolution and the limitations imposed by the presence of acoustic barriers such as air and bone.

In the current study, we successfully utilized ultrasound to determine the presence and absence of airway obstruction, based on a simple characteristic derived from the interaction of an ultrasound wavefront with an interface separating air and soft tissue. This method exploits the relatively opaque nature of this interface based on a fortified acoustic barrier set up due to the presence of air, which is deemed a very poor conductor for the transmission of sound (absence of air column = tissue apposition signifies airway collapse). The chief objective of this study of demonstrating precise airway collapsibility in a model of OSA is based on this key interaction and is reproducible. Although this was first described by 
a

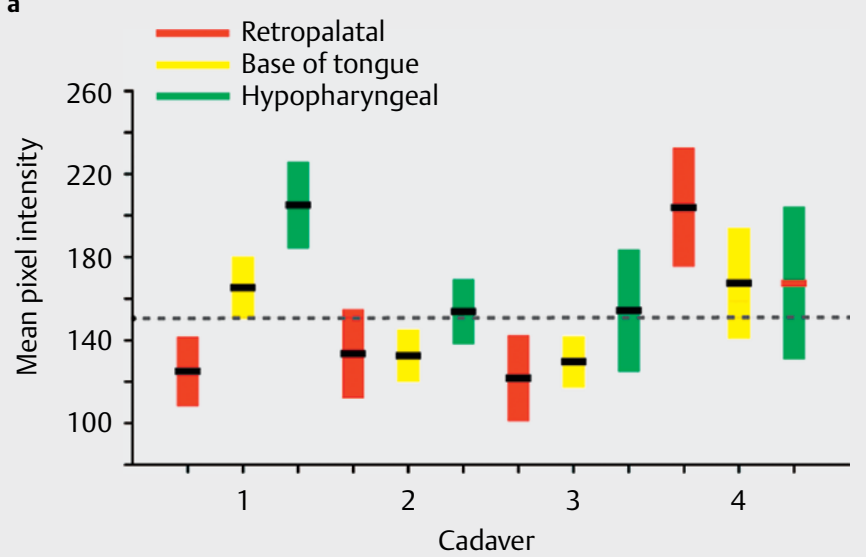

b

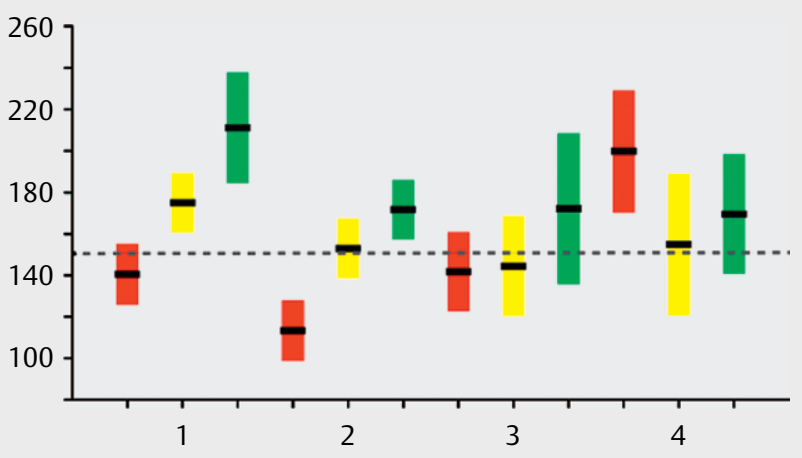

c

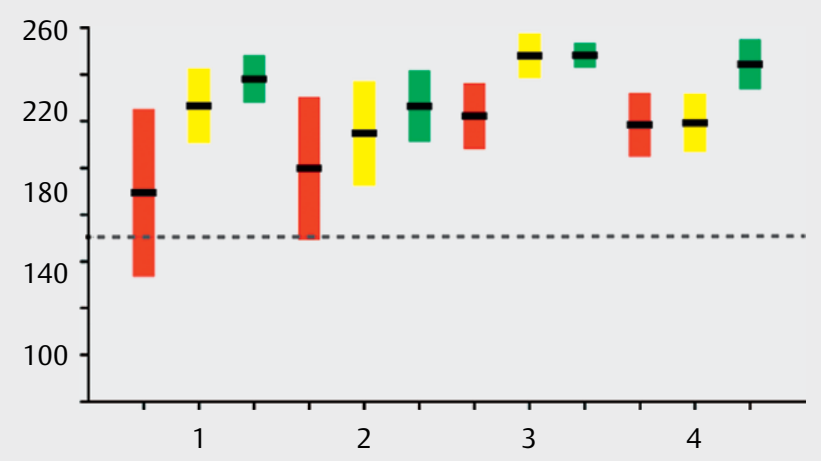

d

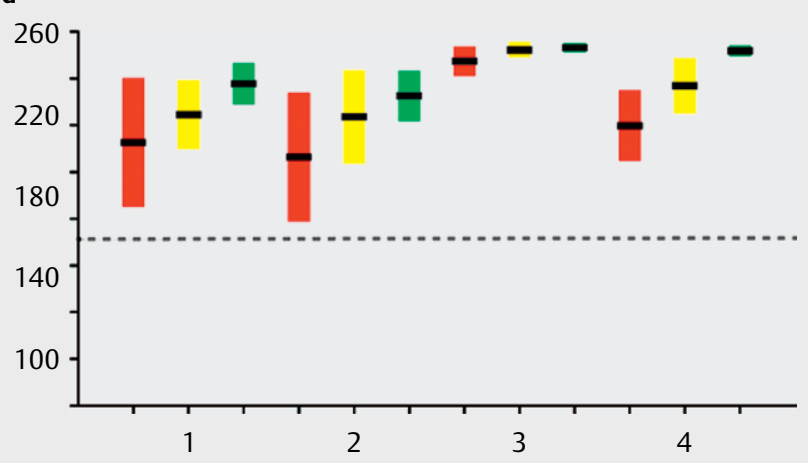

- Fig. 5 Detailed comparisons of pixel intensities from anatomic subsites within the airway, grouped by method of detection and condition. 3 anatomic subsites that include the close extents of retropalatal, base of tongue and hypopharyngeal soft tissues were analyzed. These areas corresponded to the top, middle and bottom thirds of the acquired images from each cadaver. In all 4 cadavers, the retropalatal soft tissues (red) collapsed more, leading to a recognizable drop in pixel intensity in acquired images. As is evident, the hypopharyngeal segment (green) was least prone to obstruction. A line is drawn to emphasize the empiric cut-off for intensity below which the image was considered obstructed. These plots highlight the segmental nature of airway collapse due to inherent structural differences in tissue composition, and their varied tendencies to obstruct in response to negative pressure. Rows $\mathbf{a}, \mathbf{b}$ and $\mathbf{c}, \mathbf{d}$ show data from overall conditions - open and and obstructed, whereas columns $\mathbf{a}, \mathbf{c}$ and $\mathbf{b}, \mathbf{d}$ provide data from 2 different methods of estimation - manual and automated, respectively. Boxplots are defined in the same manner as in $>$ Fig. 4 .

Shu et al. [19] by the application of submental ultrasonography to demarcate air-tissue spaces within the upper neck in patients with polysomnographically proven OSA, we theorized that precise characterization of the anatomic extent of collapse would be fraught with a significant amount of noise in the data, owing to the inherent nature of interaction between ultrasound and soft tissue. This problem is generally offset in our study by identification of the air-tissue interface alone, without reliance on calculation of the anatomic extent posteriorly, as the ultrasound wavefront may be attenuated significantly past the airway. In fact, we could not identify a single instance in which a brighter interface was found anterior to the airway, leading to consistent visualization of air-tissue interaction. More importantly, the use of a rotary transducer facilitates nearly simultaneous identification of all anatomic sites susceptible to collapse.

Our results demonstrating a discernible correlation between manually and automatically determined extents of air-tissue interaction warrant additional evaluation in human subjects. The combination of morphological operations within the context of image processing techniques used in this study was able to satisfactorily match manual labelling of images, albeit with a lack of the use of a training dataset. It may therefore be construed that the parameters were chosen arbitrarily based on what may be considered clinically apparent airway obstruction from visual inspection. Further research is necessary to determine the functional significance of obstruction measured by ultrasound by combining it with polysomnography.

We have identified several other areas within this study that need improvement. The primary limitation of our study is that the same individual was involved in visual inspection of the images as well as determination of the steps of the image processing protocol. We acknowledge that although our preliminary results appear promising, there is a need to examine the external validity of these results by additional testing in (i) a blinded fashion, and (ii) in live subjects. Additionally, the proportion of noise within our recordings may be lower than real-time assessments performed in live human subjects, as the latter may be subject to additional artifacts such as movement and swallowing. Consequently, more manual input may be obligatory to circumvent image contamination from these artifacts. Second, we could not reliably estimate the size of the air column due to the attenuation of the wavefront beyond the air-tissue interface. This may be resolved by the addition of sepa- 


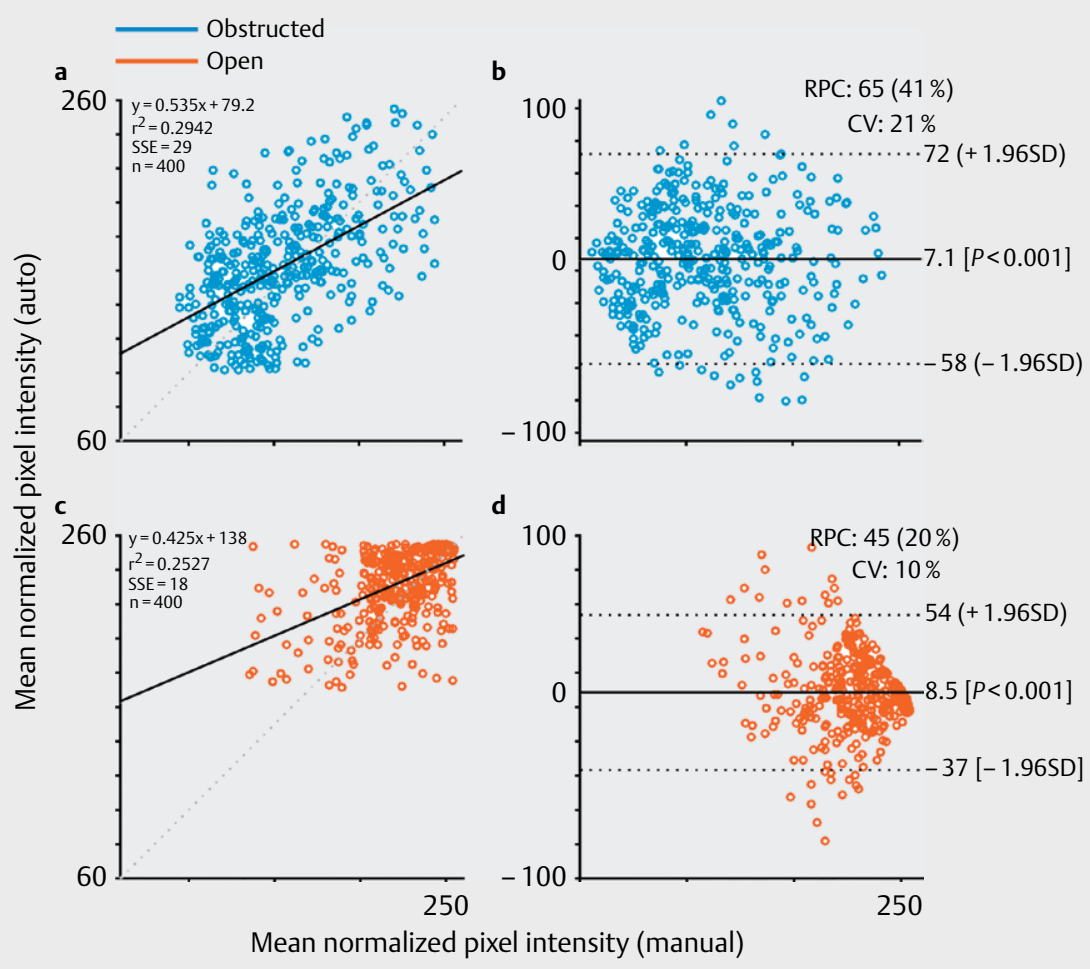

- Fig. 6 Reliability assessments using Bland-Altman plots. Each scatter diagram in a and $\mathbf{c}$ is constructed by plotting the distribution of manual estimations and automated measurements of mean pixel intensities in obstructed and open conditions, respectively. The degree of correlation was estimated using Pearson R and equations as shown in the top left. Obstructed condition correlated better as is evident from a. In b, d, the Bland-Altman plots that depict the distribution of differences between the manual and automated methods are rendered. The reproducibility coefficient (RPC) and the coefficient of variation (CV), which were both significantly higher in the obstructed condition, are within the top right portion of each panel.

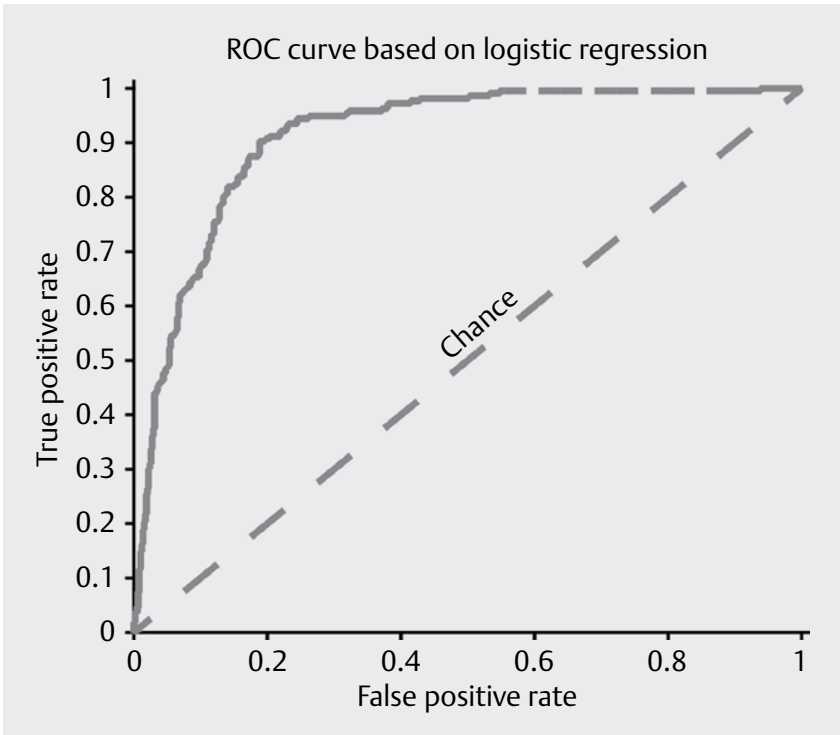

-Fig. 7 Assessment of classification ability using ROC analysis. Following logistic regression fitted to the distribution of images considered obstructed or not based on an empiric mean intensity threshold of 150 gray level units, an ROC curve was constructed, showing the variation of the true positive rate with the false positive rate. An AUC of 0.91 established the satisfactory classification achieved by this method. Chance distribution is plotted as a diagonal. rate transducers to image the sagittal plane. We also propose the use of spatial tagging to maintain uninterrupted focus on the ROI, as is used in color Doppler imaging. This may additionally increase the reliability of recordings given those circumstances. In addition, our present technique is limited with respect to volumetric assessments as the transducer does not provide information in the coronal plane. To mitigate this, the addition of an additional transducer that conforms to that plane may be necessary to provide that anatomic detail. Other transducer shapes, e. g., concave, may better conform to the shape of the neck, and may thus improve imaging precision. Importantly, our image processing technique has been validated by endoscopic assessments and visual calculations only. The lack of polysomnographic validation as performed by a previous study [18] is thus noteworthy. Although we consider the image processing techniques used in this study to be the first such application in site of obstruction imaging, they could be improved even further with the use of machine-learning algorithms such as those used in automated analysis of PSG recordings [22]. Although our results are based on relatively brief recordings (800 images in total), we expect that our techniques are likely to be more successful when prolonged recordings are available.

We envisage that this device could be used in conjunction with a pulse-oximeter trigger as a primary diagnostic modality in OLT studies. Additionally, we envisage that the foremost application of this technology would be its complementary addition to a full-panel PSG in the identification of the site of airway obstruction. Patients 
with OSA may have obstruction at various levels of the airway, including the uvula-soft palate complex, the base of tongue or hypopharyngeal sites [23]. Undoubtedly, success rates of procedures such as uvulopalatopharyngoplasty (UPPP) and tongue base reduction that address single anatomic sites may be affected by the lack of knowledge of simultaneous obstruction occurring in other sites. Conversely, multi-level surgery, which is often suggested without reliable demonstration of obstruction that prevails in all of these sites may add to unnecessary procedural morbidity. For these reasons, a review by Stuck et al. [24] showed that, despite the widespread use of the Müller maneuver and sedated endoscopy, there is minimal evidence supporting their use in improving treatment outcomes. The relative flaws associated with these various tests are addressed by ultrasonographic assessments as shown in this study, wherein functional assessments could be carried out without the introduction of instruments directly into the airway. Thus, further refinement of ultrasound technology for this use and subsequent clinical validation using PSG are likely to advance the application of ultrasound technology to the dynamic noninvasive localization of airway obstruction.

\section{Conflict of interest}

1. Jeffrey Wolf and Amal Isaiah have a patent pending (USPTO0209001) in relation to the technology described in this study and are recipients of royalty payments from the University of Maryland School of Medicine, Baltimore, MD.

2. Jeffrey Wolf has an equity position in Tesserae LLC, a sleep apnea imaging company.

\section{References}

[1] Strollo PJ], Rogers RM. Obstructive sleep apnea. N Engl J Med 1996; 334: 99-104

[2] Nieto F], Young TB, Lind BK, Shahar E, Samet JM, Redline S et al. Association of sleep-disordered breathing, sleep apnea, and hypertension in a large community-based study. Sleep Heart Health Study. JAMA 2000; 283: 1829-1836

[3] Coughlin SR, Mawdsley L, Mugarza JA, Calverley PMA, Wilding JPH. Obstructive sleep apnoea is independently associated with an increased prevalence of metabolic syndrome. Eur Heart J 2004; 25 : 735-741

[4] Findley LJ, Barth JT, Powers DC, Wilhoit SC, Boyd DG, Suratt PM. Cognitive impairment in patients with obstructive sleep apnea and associated hypoxemia. Chest 1986; 90: 686-690

[5] Partinen M, Jamieson A, Guilleminault C. Long-term outcome for obstructive sleep apnea syndrome patients. Mortality. Chest 1988; 94 : 1200-1204

[6] Monasterio C, Vidal S, Duran J, Ferrer M, Carmona C, Barbe F et al. Effectiveness of continuous positive airway pressure in mild sleep apnea-hypopnea syndrome. Am J Respir Crit Care Med 2001; 164: 939-943
[7] Epstein L], Kristo D, Strollo PJ, Friedman N, Malhotra A, Patil SP et al. Clinical guideline for the evaluation, management and long-term care of obstructive sleep apnea in adults. J Clin Sleep Med JCSM Off Publ Am Acad Sleep Med 2009; 5: 263-276

[8] Pang KP, Gourin CG, Terris DJ. A comparison of polysomnography and the WatchPAT in the diagnosis of obstructive sleep apnea. Otolaryngol-Head Neck Surg Off J Am Acad Otolaryngol-Head Neck Surg 2007; 137: 665-668

[9] Chiner E, Signes-Costa J, Arriero JM, Marco J, Fuentes I, Sergado A. Nocturnal oximetry for the diagnosis of the sleep apnoea hypopnoea syndrome: a method to reduce the number of polysomnographies? Thorax 1999; 54: 968-971

[10] Rama AN, Tekwani SH, Kushida CA. Sites of obstruction in obstructive sleep apnea. Chest 2002; 122: 1139-1147

[11] Shepard JW], Thawley SE. Localization of upper airway collapse during sleep in patients with obstructive sleep apnea. Am Rev Respir Dis 1990; 141: 1350-1355

[12] Lowe AA, Fleetham JA, Adachi S, Ryan CF. Cephalometric and computed tomographic predictors of obstructive sleep apnea severity. Am J Orthod Dentofac Orthop Off Publ Am Assoc Orthod Its Const Soc Am Board Orthod 1995; 107: 589-595

[13] Schwab RJ, Pasirstein M, Pierson R, Mackley A, Hachadoorian R, Arens $R$ et al. Identification of upper airway anatomic risk factors for obstructive sleep apnea with volumetric magnetic resonance imaging. Am J Respir Crit Care Med 2003; 168: 522-530

[14] Sustic A. Role of ultrasound in the airway management of critically ill patients. Crit Care Med 2007; 35: S173-S177

[15] Solbiati L, Osti V, Cova L, Tonolini M. Ultrasound of thyroid, parathyroid glands and neck lymph nodes. Eur Radiol 2001; 11: 2411-2424

[16] Prasad A, Yu E, Wong DT, Karkhanis R, Gullane P, Chan VWS. Comparison of sonography and computed tomography as imaging tools for assessment of airway structures. J Ultrasound Med Off J Am Inst Ultrasound Med 2011; 30: 965-972

[17] Or DYL, Karmakar MK, Lam GCS, Hui JWY, Li JW, Chen PP. Multiplanar 3D ultrasound imaging to assess the anatomy of the upper airway and measure the subglottic and tracheal diameters in adults. $\mathrm{Br}$ J Radiol 2013; 86: 20130253

[18] Chen J-W, Chang C-H, Wang S-J, Chang Y-T, Huang C-C. Submental ultrasound measurement of dynamic tongue base thickness in patients with obstructive sleep apnea. Ultrasound Med Biol 2014; 40: 2590-2598

[19] Shu C-C, Lee P, Lin J-W, Huang C-T, Chang Y-C, Yu C-J et al. The use of sub-mental ultrasonography for identifying patients with severe obstructive sleep apnea. PloS One 2013; 8: e62848

[20] Aldrich JE. Basic physics of ultrasound imaging. Crit Care Med 2007; 35: S131-S137

[21] Dempsey JA, Veasey SC, Morgan BJ, O’Donnell CP. Pathophysiology of sleep apnea. Physiol Rev 2010; 90: 47-112

[22] Polat K, Yosunkaya S, Gunes S. Comparison of different classifier algorithms on the automated detection of obstructive sleep apnea syndrome. J Med Syst 2008; 32: 243-250

[23] Crumley RL, Stein M, Gamsu G, Golden J, Dermon S. Determination of obstructive site in obstructive sleep apnea. The Laryngoscope 1987; 97: 301-308

[24] Stuck BA, Maurer JT. Airway evaluation in obstructive sleep apnea. Sleep Med Rev 2008; 12: 411-436 